\title{
Optimization of asymmetric figure-8 undulator as circularly polarized light source
}

\author{
Katsutoshi Shirasawa* and Atsunari Hiraya \\ Department of Physical Science, Hiroshima University, 1-3-1 Kagamiyama, Higashi-Hiroshima, Hiroshima 739-8526, Japan
}

Takashi Tanaka and Hideo Kitamura

SPring-8/RIKEN, 1-1-1 Kouto, Mikazuki-cho, Sayo-gun, Hyogo 679-5148, Japan

(Received 1 December 2003; published 27 February 2004)

\begin{abstract}
In the soft x-ray region, the demand for helicity switching of circularly polarized undulator radiation is increasing to study circular dichroism. The asymmetric figure- 8 undulator is an insertion device to provide circularly polarized radiation (CPR) and a simple method of helicity switching which has been proposed and built at SPring-8. Because of its complicated magnetic structure, it is important to optimize the magnetic parameters for more effective utilization of CPR. Using an analytical method, the relation between the magnetic parameters to maximize the degree of circular polarization is obtained. The impact of the finite emittance of electron beams on the polarization performance is also investigated numerically.
\end{abstract}

DOI: 10.1103/PhysRevSTAB.7.020702

PACS numbers: 41.60.Ap

\section{INTRODUCTION}

Helicity switching of circularly polarized undulator radiation provides a valuable tool in synchrotron radiation experiments, particularly in the study of circular dichroism. Since there are no practical phase retarders in the soft $\mathrm{x}$-ray region, the light source should have a function to switch the helicity. Therefore, various insertion devices (IDs), which can switch the helicity of circularly polarized radiation, have been proposed and built [1-6].

These devices can be classified into two types. One is changing the helicity of the magnetic field in a helical undulator by a mechanical shift (phasing) of magnet arrays $[1,2]$ or by reversing the polarity of electromagnets $[1,3,4]$. Since right and left circular photon beams are emitted from the same undulator, photon sources of two polarized states are ideally identical. However, the eddy current in a vacuum chamber induced by rapid reverse of the undulator field disturbs the stability of electron beam orbits which should be corrected by a feedback system.

The second way for helicity switching is to use two helical undulators with opposite helicity. The electron beam is either displaced for two parallel trajectories [5] or deflected by a chicane [6] in each undulator and the photon beam is chopped by a chopper installed in the beam line. Since the chopper is independent of the electron beam, there is no effect on a storage ring operation and the switching frequency can be increased. At SPring-8, right and left circular polarizations (RCP and LCP) are alternately supplied to the beam line by switching two horizontal bumps of the electron orbit generated by kicker magnets. In this method, RCP and LCP are emitted on the same axis; therefore the distributions of two beams are approximately the same in horizontal

*Electronic address: kshira@spring8.or.jp phase space and partially overlapped in vertical phase space [7].

We have recently constructed an ID composed of permanent magnets and electromagnets [8]. It is similar to the figure-8 undulator installed in SPring-8 [9]; the side arrays consist of normal permanent magnets, while the central array consists of electromagnets to change the operation mode. The horizontal field is produced by permanent magnets and tuned by moving them along the undulator axis. On the other hand, the vertical field is produced by the electromagnets and tuned by changing coil current. Thanks to the tunability of the electromagnets, the device has three operation modes: figure-8, helical, and asymmetric figure- 8 modes [10]. Among these modes, the asymmetric figure- 8 mode provides a simple method of helicity switching. Using an appropriate combination of magnetic parameters, pure circularly polarized radiation (CPR) is obtained at certain harmonics. In particular, the 1.5th and 2 nd harmonics are promising from the viewpoint of fast helicity switching. It is therefore important to investigate the polarization properties of the 1.5th and 2nd harmonics and optimize the magnetic parameters of the asymmetric figure- 8 undulator, which are the main subjects of this article.

\section{ASYMMETRIC FIGURE-8 UNDULATOR}

\section{A. Principle}

The asymmetric figure- 8 undulator has been proposed as a multipolarization light source with low on-axis power density [11]. It is based on a modification of the figure-8 undulator [12] which provides both horizontal and vertical polarizations. Figure 1 shows electron orbits of the asymmetric figure- 8 undulator projected onto a transverse plane. Each trajectory of Fig. 1 generates opposite helicity of CPR to the other. The magnetic fields 

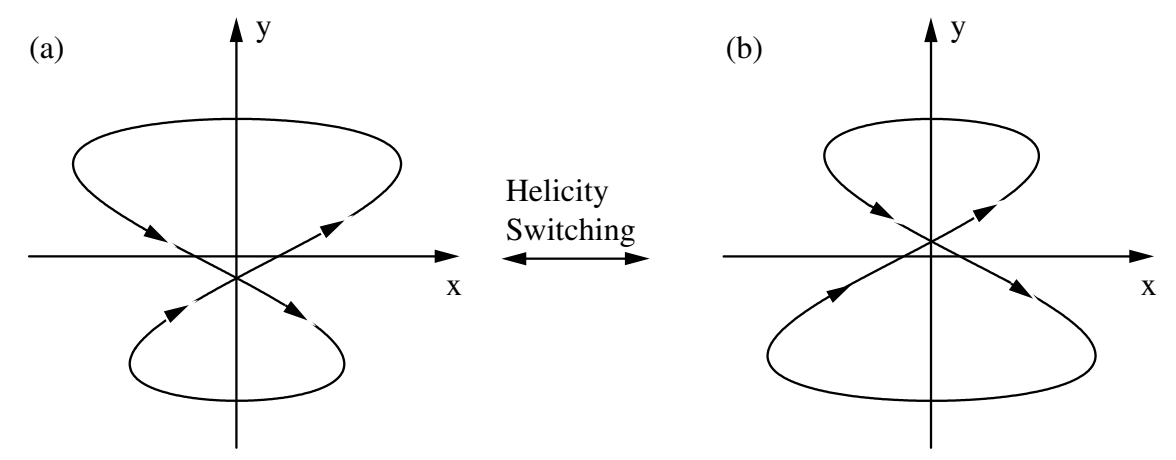

FIG. 1. The electron orbits of an asymmetric figure-8 undulator projected onto the transverse plane. In the asymmetric figure-8 undulator, the helicity switching means to change the trajectory (a) and (b).

to realize the asymmetric figure- 8 trajectory are

$$
\begin{gathered}
B_{x}=-B_{0 x} \sin \left(\pi z / \lambda_{u}\right), \\
B_{y}=B_{y 1}+B_{y 2}, \\
B_{y 1}=B_{1 y} \sin \left(2 \pi z / \lambda_{u}\right), \\
B_{y 2}=B_{2 y} \cos \left(\pi z / \lambda_{u}\right),
\end{gathered}
$$

where $\lambda_{u}$ is the period length and $B_{0 x, 1 y, 2 y}$ are the peak amplitudes of the magnetic fields. The vertical field $B_{y}$ comprises two components $B_{y 1}$ and $B_{y 2} . B_{y 1}$ and $B_{x}$ form the magnetic fields of a normal figure- 8 undulator. $B_{y 2}$ is an additional field to generate a CPR component.

The wavelength of the $k$ th harmonic on axis, $\lambda_{k}$, is given by

$$
\lambda_{k}=\frac{\lambda_{u}}{2 k \gamma^{2}}\left(1+\frac{K^{2}}{2}\right)
$$

with

$$
\begin{gathered}
K^{2}=K_{x}^{2}+K_{y 1}^{2}+K_{y 2}^{2}, \\
K_{x}=\frac{e B_{0 x} \lambda_{u}}{\pi m c}, \\
K_{y 1}=\frac{e B_{1 y} \lambda_{u}}{2 \pi m c}, \\
K_{y 2}=\frac{e B_{2 y} \lambda_{u}}{\pi m c},
\end{gathered}
$$

where $m, e, \gamma$, and $c$ are the electron's rest mass, unit charge, Lorentz factor, and the speed of light in vacuum, respectively. The flux density of the $k$ th harmonic observed on axis is [11]

$$
\frac{d^{2} P_{k}}{d \Omega d \omega}=\frac{e^{2} \gamma^{2} N^{2}}{4 \pi \varepsilon_{0} c}\left(\left|f_{k x}\right|^{2}+\left|f_{k y}\right|^{2}\right),
$$

where $N$ is the number of the undulator period, and

$$
k=\frac{1}{2}, 1, \frac{3}{2}, 2, \cdots
$$

Here $f_{k x, k y}$ are the complex field amplitudes of radiation in the horizontal and vertical directions, and they can be expressed as

$$
\begin{aligned}
& f_{k x}=\frac{2 k \xi}{\pi}\left(-Q_{1}\right) P_{N}, \\
& f_{k y}=\frac{2 k \xi}{\pi}\left(-Q_{2}\right) P_{N},
\end{aligned}
$$

with

$$
\begin{aligned}
& \xi=\frac{1}{1+K^{2} / 2} \\
& P_{N}=\frac{\sin \left(\pi N \omega / \omega_{1}\right)}{\pi N\left(k-\omega / \omega_{1}\right)}, \\
& \omega_{1}=\frac{2 \pi c}{\lambda_{1}} \\
& Q_{1}=\left\{\begin{array}{c}
\int_{0}^{2 \pi}\left(K_{y 1} \cos 2 \eta-K_{y 2} \sin \eta\right) \cos 2 k \psi d \eta \\
k=\text { integer, } \\
-i \int_{0}^{2 \pi}\left(K_{y 1} \cos 2 \eta-K_{y 2} \sin \eta\right) \sin 2 k \psi d \eta \\
k=\text { half-odd integer, }
\end{array}\right. \\
& Q_{2}=\left\{\begin{array}{c}
-i \int_{0}^{2 \pi} K_{x} \cos \eta \sin 2 k \psi d \eta \\
k=\text { integer } \\
\int_{0}^{2 \pi} K_{x} \cos \eta \cos 2 k \psi d \eta \\
k=\text { half-odd integer, }
\end{array}\right. \\
& \psi=\eta-\xi K_{y 1} K_{y 2} \cos \eta+\frac{\xi\left(K_{x}^{2}-K_{y 2}^{2}\right)}{4} \sin 2 \eta \\
& +\frac{\xi K_{y 1} K_{y 2}}{3} \cos 3 \eta+\frac{\xi K_{y 1}^{2}}{8} \sin 4 \eta,
\end{aligned}
$$

The degree of circular polarization $\left(P_{C}\right)$ of the $k$ th harmonic is calculated as 


$$
P_{C}=\left|\frac{S_{3}}{S_{0}}\right|=\left|\frac{2 \operatorname{Im} f_{k x}^{*} f_{k y}}{\left|f_{k x}\right|^{2}+\left|f_{k y}\right|^{2}}\right| \text {. }
$$

The figure of merit $(F)$, which is important for the experiments utilizing CPR, is defined as follows:

$$
F=P_{C} \sqrt{\left|f_{k x}\right|^{2}+\left|f_{k y}\right|^{2}} .
$$

As shown in the above equations, the optical properties of radiation from the asymmetric figure- 8 undulator depend on the three $K$ values, which should be optimized for higher degrees of polarization by means of analytical or numerical methods.

\section{B. Advantages}

The asymmetric figure- 8 undulator has a lot of advantages for helicity switching of circularly polarized light as follows.

(1) Fast helicity switching

The magnetic field necessary for producing circular polarization is small. Therefore, faster switching of helicity and suppression of the eddy current are expected.

(2) Low on-axis power density

The heat load on optical elements is kept low and almost constant during the helicity change.

(3) Small photon flux variation

Since the variation of the $K$ value is not large during the transition time of the helicity switching, the photon flux variation is small.

(4) Identical photon sources

Because RCP and LCP are generated by the same undulator, two photon sources are identical in phase space, i.e., the same incidence angle and intensity at the sample point are expected. This is important for user experiments.

In order to take full advantages of the asymmetric figure-8 undulator described above, it is necessary to use it with optimum magnetic fields.

\section{OPTIMIZATION}

First, the magnetic field is optimized for the zero emittance electron beam. Next, numerical simulations are performed to investigate the influence of the electron beam emittance on $P_{C}$. Finally, the figure of merit obtained from the optimized asymmetric figure- 8 undulator is shown.

\section{A. Zero emittance case}

The degree of circular polarization of the asymmetric figure-8 undulator depends on $K_{y 2}$. For example, Fig. 2 shows the dependence of $P_{C}$ on $K_{y 2} / K_{y 1}$ for each harmonic. The $K$ parameters are $K=5.4, K_{x}=3.4$, and $\sqrt{K_{y 1}^{2}+K_{y 2}^{2}}=4.2$. When $K_{y 2}=0$, the device works as a normal figure-8 undulator. Though higher $P_{C}$ of each

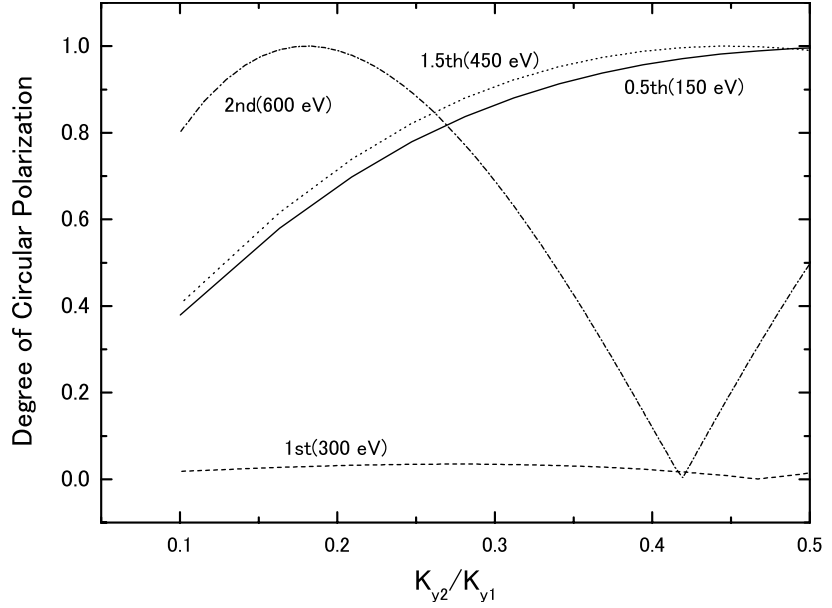

FIG. 2. $K_{y 2} / K_{y 1}$ dependence of $P_{C}$. Each line shows the different harmonics from the 0.5 th to the 2 nd. The $K$ and $K_{x}$ are fixed at $K=5.4$ and $K_{x}=3.4$. The fundamental photon energy is $300 \mathrm{eV}$.

harmonic is obtained for larger $K_{y 2} / K_{y 1}$, smaller $K_{y 2}$ is desirable for fast helicity switching.

It is convenient to introduce new parameters defined as

$$
\left(\begin{array}{c}
\phi=\tan ^{-1}\left(\frac{K_{y 2}}{K_{y 1}}\right), \\
\theta=\tan ^{-1}\left(\frac{\sqrt{K_{y 1}^{2}+K_{y 2}^{2}}}{K_{x}}\right) .
\end{array}\right.
$$

Figure 3 shows the geometrical definitions of $\theta$ and $\phi$. Let us look for the condition to maximize $P_{C}$, i.e., $S_{0}=S_{3}$. From Eqs. (11), (12), and (19), we can rewrite the above condition as

$$
\left|Q_{1}\right|^{2}+\left|Q_{2}\right|^{2}=2 \operatorname{Im}\left(Q_{1}^{*} Q_{2}\right) .
$$

After substituting Eq. (21) into (16) and (17), we find that the variable $K$ vanishes from Eq. (22), meaning that the optimum relation between $\theta$ and $\phi$ is independent of $K$ or the fundamental photon energy.

Figure 4(a) shows the relation between $\theta$ and $\phi$ to obtain $P_{C}=1$ with the 1.5 th and 2 nd harmonics. While $P_{C}=1$ can be obtained with any $\theta$ in Fig. 4(a), the intensity has a maximum at a specific value of $\theta$ and therefore $\phi$. Figure $4(\mathrm{~b})$ shows $F, P_{C}$ times intensity, calculated for $\theta$ and $\phi$ in Fig. 4(a).

\section{B. Real emittance case}

Having described the optimization with the zero emittance electron beam, let us now consider the influence of the finite electron beam emittance on $P_{C}$. The synchrotron radiation calculation code, SPECTRA, developed at the SPring-8 [13], has been used for numerical computation. The electron beam parameters of SPring- 8 shown in Table I are used in the calculations. The device is assumed to have the period length $\left(\lambda_{u}\right)$ of $13 \mathrm{~cm}$ and the total length of $4.16 \mathrm{~m}$. 


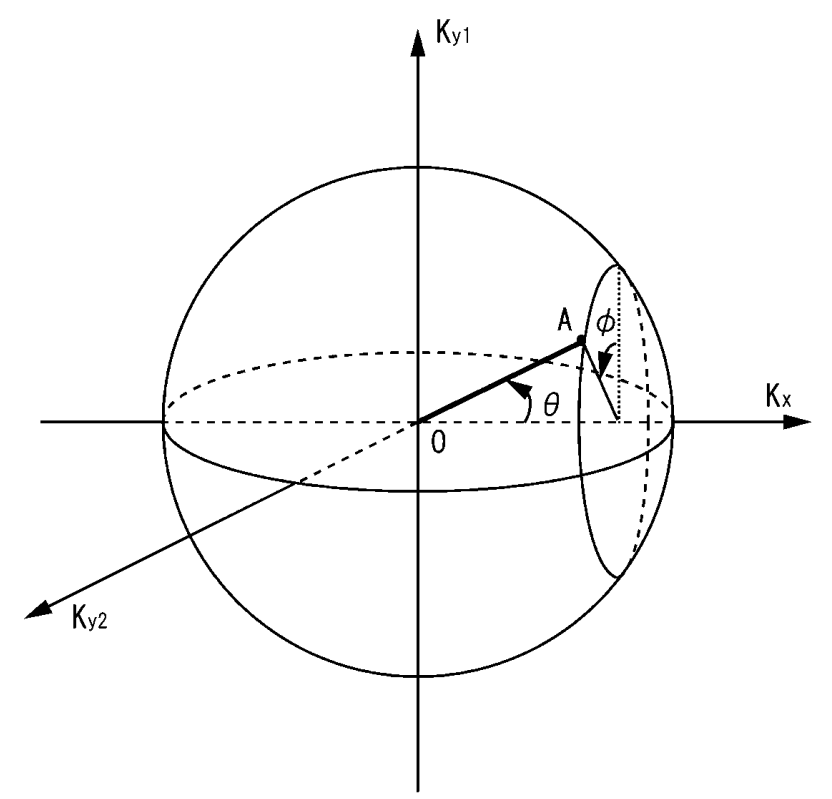

FIG. 3. The geometrical definition of $\theta$ and $\phi$. The length $|O A|$ presents $K$. The angles $\theta$ and $\phi$ are determined by $K_{y 1}$, $K_{y 2}$, and $K_{x}$.

Figure 5 shows (a) $P_{C}$ and (b) $F$ calculated using the optimized parameters for the 1.5th and 2nd harmonics. As shown in Fig. 5, $P_{C}$ is degraded due to the electron beam emittance. In the 2 nd harmonic, $P_{C}$ becomes almost maximum at $\theta=45^{\circ}$ for all photon energies, from

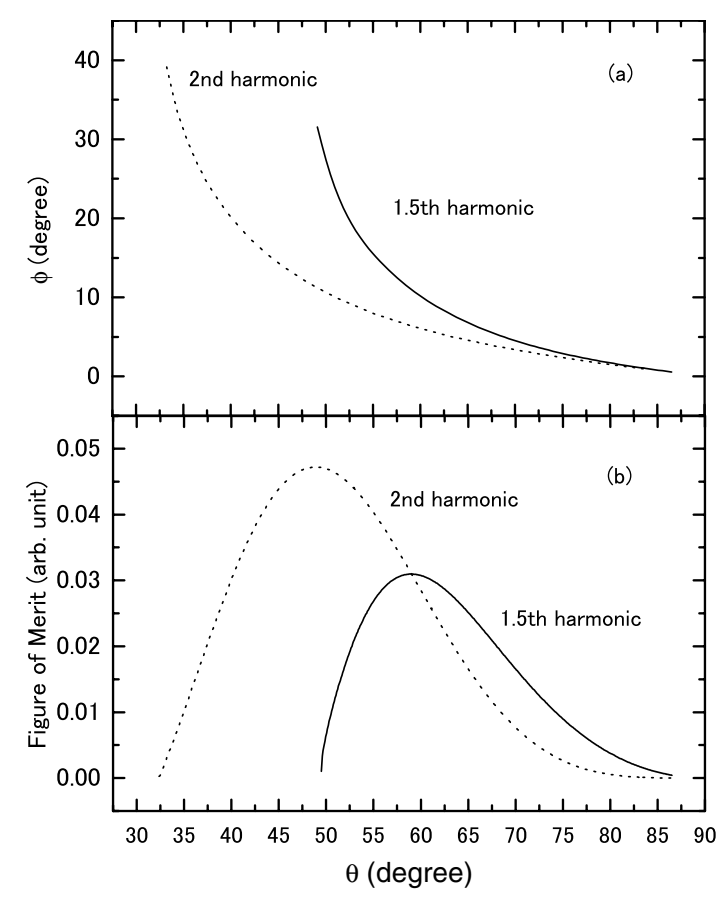

FIG. 4. (a) $\theta$ and $\phi$ to obtain $P_{C}=1$. (b) Calculated $F$ using the parameters shown in (a). The solid lines show the 1.5th harmonic and the dotted lines show the 2 nd harmonic.
TABLE I. The electron beam parameters used in the calculations.

\begin{tabular}{lc}
\hline \hline Energy & $8 \mathrm{GeV}$ \\
Average current & $100 \mathrm{~mA}$ \\
Natural emittance & $5.9 \times 10^{-9} \mathrm{~m} \mathrm{rad}$ \\
Coupling constant & $0.3 \%$ \\
Horizontal beta function & $24.35 \mathrm{~m}$ \\
Vertical beta function & $5.77 \mathrm{~m}$ \\
Energy spread & $0.11 \%$ \\
\hline \hline
\end{tabular}

200 to $2000 \mathrm{eV}$, and optimum $\theta$ for the 1.5 th harmonic is $65^{\circ}$.

Since the 2nd harmonic shows always higher $P_{C}$ and $F$ than the 1.5 th harmonic under optimized conditions, we use the 2nd harmonic of the asymmetric figure- 8 undulator with $\theta=45^{\circ}$ as a circularly polarized light source. The reason why $P_{C}$ is so sensitive to the electron beam emittance is explained in Fig. 6, which shows the spatial distribution of the Stokes parameter $S_{3}$ for the 2nd harmonic calculated with the zero emittance beam. The parameters $K_{x}, K_{y 1}$, and $K_{y 2}$ are assumed to satisfy the optimum conditions shown in Fig. 4(a) and the fundamental photon energy is fixed at $400 \mathrm{eV}$. When $\theta=68^{\circ}$, two regions with opposite helicity $\left(S_{3}>0\right.$ at left and $S_{3}<0$ at right) are close to each other, and it will degrade $P_{C}$ when taking into account the electron beam emit-

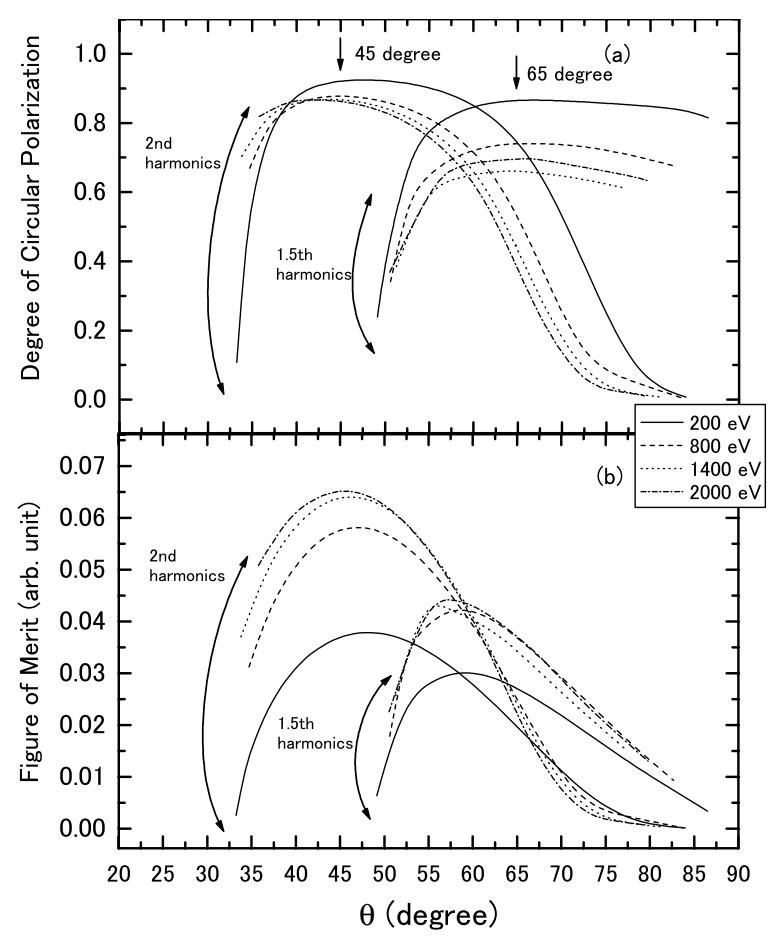

FIG. 5. (a) $P_{C}$ and (b) $F$ at the 2 nd and 1.5 th harmonics calculated using the optimized parameters shown in Fig. 4(a). These calculations take into account the electron beam emittance. 


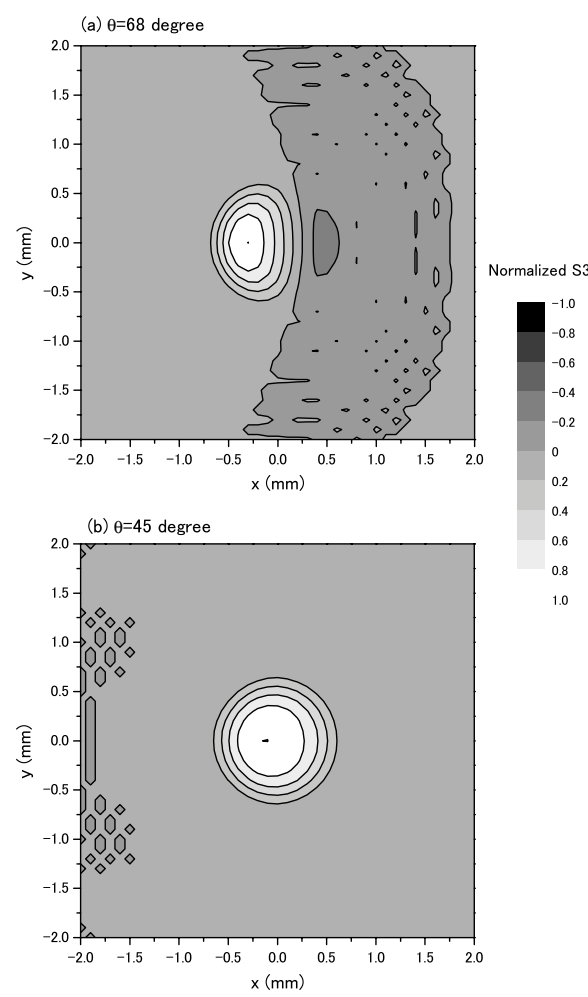

FIG. 6. The spatial distributions of $S_{3}$ of the 2nd harmonic with (a) $\theta=68^{\circ}$ and (b) $\theta=45^{\circ}$. The fundamental photon energy is $400 \mathrm{eV}$ and the screen position is $30 \mathrm{~m}$ away from the light source. The helicities of the white- and black-colored regions are opposite.

tance. On the other hand, most of the photons have the same helicity $\left(S_{3}>0\right)$ when $\theta=45^{\circ}$. Thus the degradation of $P_{C}$ is small compared with the case of $\theta=68^{\circ}$.

\section{SUMMARY}

The magnetic field of the asymmetric figure- 8 undulator was optimized as a circularly polarized light source in the soft x-ray region (200-2000 eV). From the numerical analyses, it was found that the electron beam emittance significantly affects $P_{C}$. With considering the emittance effect, the 2 nd harmonic with $\theta=45^{\circ}$ was found to be the best solution to obtain high $P_{C}$ for a wide energy range in the asymmetric figure- 8 undulator. The 1.5th harmonic was also optimized, but $P_{C}$ and $F$ were lower than those of the 2 nd harmonic. We can simply obtain the optimum magnetic field parameters by using the above results.

[1] H. Onuki, Nucl. Instrum. Methods Phys. Res., Sect. A 246, 94 (1986).

[2] A. Agui, A. Yoshigoe, T. Nakatani, T. Matsushita, Y. Saitoh, A. Yokoya, H. Tanaka, Y. Miyahara, T. Shimada, M. Takeuchi, T. Bizen, S. Sasaki, M. Takao, H. Aoyagi, T. P. Kudo, K. Satoh, S. Wu, and H. Ohkuma, Rev. Sci. Instrum. 72, 3191 (2001).

[3] R. P. Walker and B. Diviacco, Rev. Sci. Instrum. 63, 332 (1992).

[4] J. Chavanne, P. Elleaume, and P. Vaerenbergh, J. Synchrotron Radiat. 5, 196 (1998).

[5] T. Schmidt, G. Ingold, A. Imhof, B. D. Patterson, L. Patthey, C. Quitmann, C. Schulze-Briese, and R. Abela, Nucl. Instrum. Methods Phys. Res., Sect. A 467-468, 126 (2001).

[6] M. Weiss et al., Nucl. Instrum. Methods Phys. Res., Sect. A 467-468, 449 (2001).

[7] T. Hara, K. Shirasawa, M. Takeuchi, T. Seike, Y. Saito, T. Muro, and H. Kitamura, Nucl. Instrum. Methods Phys. Res., Sect. A 498, 496 (2003).

[8] K. Shirasawa, T. Tanaka, T. Seike, A. Hiraya, and H. Kitamura, in Proceedings of the 8th International Conference on Synchrotron Radiation Instrumentation (to be published).

[9] T. Tanaka, T. Hara, M. Oura, H. Ohashi, H. Kimura, S. Goto, Y. Suzuki, and H. Kitamura, Rev. Sci. Instrum. 70, 4153 (1999).

[10] T. Tanaka, K. Shirasawa, and H. Kitamura, Rev. Sci. Instrum. 73, 1724 (2002).

[11] T. Tanaka and H. Kitamura, Nucl. Instrum. Methods Phys. Res., Sect. A 449, 629 (2000).

[12] T. Tanaka and H. Kitamura, Nucl. Instrum. Methods Phys. Res., Sect. A 364, 368 (1995).

[13] T. Tanaka and H. Kitamura, J. Synchrotron Radiat. 8, 1221 (2001). 